\title{
The role of timbre in the segregation of simultaneous voices with intersecting $F_{0}$ contours
}

\author{
JOHN F. CULLING and C. J. DARWIN \\ University of Sussex, Brighton, England
}

\begin{abstract}
When the fundamental frequency $\left(F_{0}\right)$ contours of two speakers' voices intersect, the listener is presented with a problem. The listener must decide which of the $F_{0}$ contours emerging from the intersection is a continuation of which contour entering the intersection: have the $F_{0}$ contours crossed or merely approached and parted? In the present experiment, subjects listened to two simultaneous diphthong-like sounds with $F_{0}$ contours that either approached and diverged or crossed over. The task was to report whether the pitches "crossed" or "bounced" away from each other. Despite the changing timbres of the two sounds, the subjects were able to discriminate crossing and bouncing $F_{0} \mathrm{~s}$, provided that the timbres of the vowels differed at the moment when their $F_{0}$ s were the same. When the timbres were the same, the subjects could not make the discrimination and tended to hear a bouncing percept. These results are consistent with the idea that listeners use continuity of timbre rather than continuity of $F_{0}$ movement to disambiguate $F_{0}$ intersections.
\end{abstract}

A number of studies have demonstrated the importance of fundamental frequency $\left(F_{0}\right)$ differences in the perceptual separation of competing voices with constant $F_{0} s$. These studies have shown that listeners can recognize the content of the speech more reliably when the $F_{0}$ s of the two voices differ than when they are identical. This result was first shown by using monotonic resynthesis from a linear predictive coding (Brokx \& Nooteboom, 1982) and was subsequently explored in detail by using steadystate synthesized vowels (Assmann \& Summerfield, 1990; Chalikia \& Bregman, 1989; Darwin \& Culling, 1990; Scheffers, 1983; Zwicker, 1984).

Parsons (1976) described an algorithm that was intended to separate the speech of two simultaneous voices by using $F_{0}$ differences. The algorithm identified two $F_{0} s$ in each analysis frame and selected components of the Fourier spectrum that corresponded to harmonics of each $F_{0}$. Parsons found that the exploitation of $F_{0}$ differences for separating voices is complicated by changes in $F_{0}$, so his speaker-separation algorithm included heuristics to help assign $F_{0}$ s arising from the same voice to the same output channel from one frame to the next. One such heuristic was that the pitches of each speaker "do not change radically" from frame to frame. Further, the algorithm extrapolated from the changes in pitch of each speaker in the last two frames to predict the changes in the next frame. The new pitches were assigned so as to match those

The authors would like to thank Quentin Summerfield and two reviewers for valuable comments on earlier versions of this paper. Address correspondence to J. F. Culling, MRC Institute of Hearing Research, University of Nottingham, University Park, Nottingham, NG7 2RD, UK. predicted as closely as possible. This algorithm thus had much in common with the Gestalt grouping principle of "good continuity," and could track the $F_{0} \mathrm{~s}$ of each speaker correctly, even when the $F_{0}$ contours crossed.

Because listeners exploit differences in $F_{0}$, the present experiment addresses the question of how listeners deal with $F_{0}$ movements across $F_{0}$ intersections. In particular, when two $F_{0}$ trajectories meet, the listener must discern whether the $F_{0} \mathrm{~s}$ cross or merely make a close approach and then diverge. Bregman, Halpern, and Chalikia (1982) conducted psychophysical experiments that examined the role of Gestalt grouping principles at $F_{0}$ intersections. They investigated (1) the role of good continuity of pitch movement; (2) the role of good continuity of timbre; and (3) the relative roles of the common fates of $F_{0} s$ and individual harmonics.

Bregman et al.'s (1982) subjects were pretrained in the recognition of four pure-tone frequency contours: a rise, a fall, a rise-fall, and a fall-rise. In their experiment, they employed pairs of pure or complex tones; one rising and one falling in pitch, which were presented concurrently and repeatedly. The subjects' task was to rank the similarity of the four frequency contours used in training to their perception of the pitch contours of the two tones. Rankings of the two uninflected pitch contours (steady rise/fall) were taken as an index of the perception of "crossing" pitch contours, whereas ratings of the two inflected contours (fall-rise/rise-fall) were taken to indicate the perception of "bouncing" pitch contours, which converged and then diverged away from each other.

In the case of two pure tones intersecting midway through the stimulus, subjects' perceptions were heavily biased toward the perception of bouncing, suggesting that 
proximity of pitch before and after the intersection was a stronger grouping principle than continuity of pitch change through the intersection. Of particular interest to the present study were the results obtained by using two complex tones. Each tone contained three equal-amplitude components and the two tones had either the same or different timbres; each tone contained either the 3rd, 4th, and 5th harmonics or the 10th, 11th, and 12th.

Bregman et al.'s (1982) three main conditions are illustrated in Figure 1. When the complexes had different timbres (middle panel), high rankings were given to the rise/fall (crossing) contours, but if the timbres were the same (top panel), rise-fall/fall-rise (bouncing) contours were ranked slightly higher; the two pitch contours were perceived to rebound from each other at the point of contact, rather than crossing over.

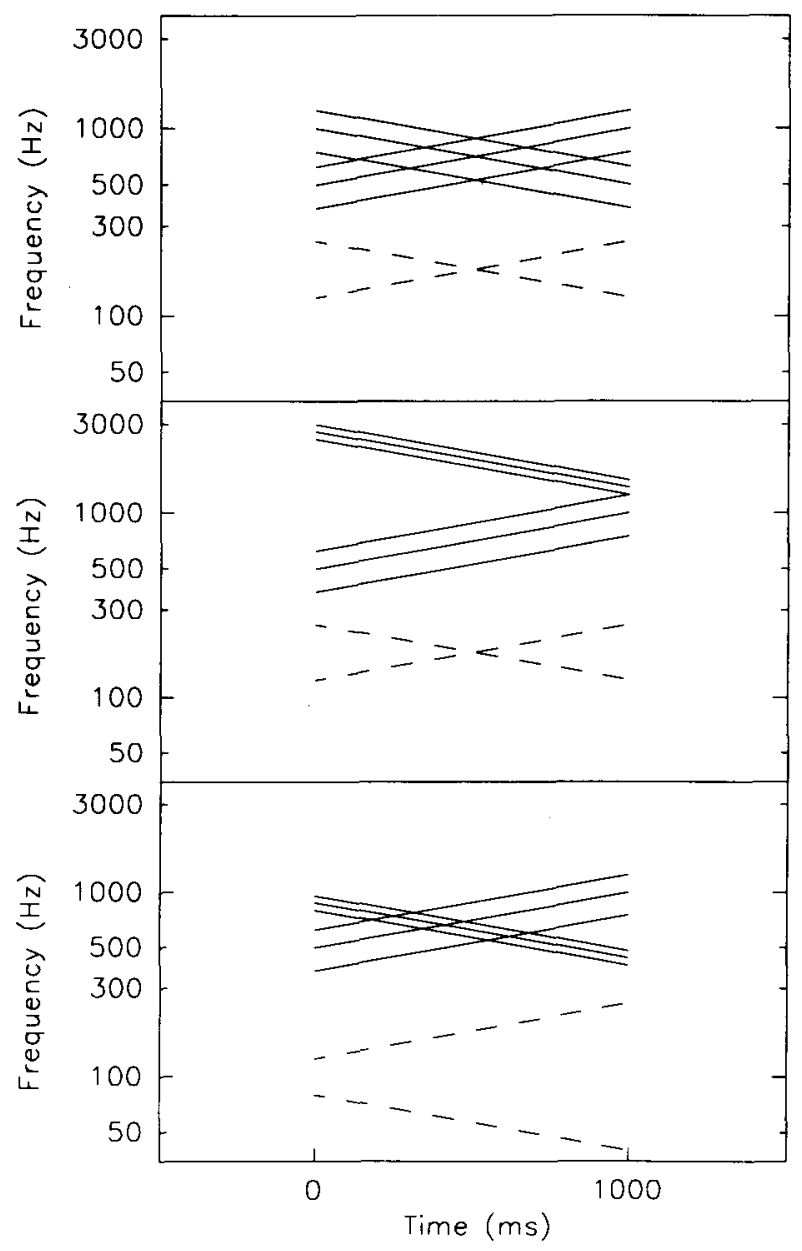

Figure 1. Illustration of the movements of $F_{0} s$ (dashed lines) and frequency components (solid lines) in the three main conditions $(\mathrm{D}$, $E$, and $F$ ) of Halikia, Bregman, and Halpern (1982). The $F_{0}$ values are estimated from Figure 1 of that paper. (From "Auditory partitioning of a set of tonal glides" by A. S. Bregman, L. Halpern, \& M. H. Chalikia, 1982, unpublished manuscript. Adapted by permission of authors.)
Bregman et al.'s (1982) findings suggest that continuity of timbre can be used to disambiguate an $F_{0}$ crossing. If the auditory system uses continuity of $F_{0}$ movement at $F_{0}$ intersections, then "crossing" judgments should have dominated in all conditions, because a "bouncing" judgment necessarily indicates the perception of an inflected pitch contour. In fact, crossing judgments predominated only when the timbres of the two sounds differed, whereas for sounds of identical timbre (in which continuity of timbre offers no cues toward either percept), bouncing judgments were slightly more frequent than crossing judgments. Good continuity of two different timbres may, therefore, be used in order to distinguish between occasions when $F_{0}$ contours cross over and when they merely make a close approach.

However, in the different-timbre condition, the individual harmonics did not cross, raising the possibility that it is the crossing of individual harmonics that somehow gives rise to the bouncing percept when the timbres are the same. A third condition addressed this issue; the $F_{0}$ of one tone was lower, so that its 10th, 11th, and 12th harmonics intersected with the $3 \mathrm{rd}, 4$ th, and 5 th harmonics of the other tone, but its $F_{0}$ did not cross that of the other tone (see Figure 1, bottom panel). Here, the subjects judged the stimuli to be crossing slightly less than when the $F_{0} \mathrm{~s}$ crossed and the harmonics did not, but crossing responses still dominated. The intersection of individual harmonics is unlikely, therefore, to have been responsible for producing the bouncing percept when the timbres were the same.

The present experiment extended and refined Bregman et al.'s (1982) experiment to overcome two possible confounding influences:

1. Continuity of timbre may have been confounded by the common fate of the individual harmonics. When sounds had the same timbres (Figure 1, top panel), the corresponding components of each sound crossed each other simultaneously at the same time that the $F_{0}$ s crossed, making their behavior at the intersection similarly ambiguous at that moment. Indeed, the individual harmonics of these stimuli undergo many intersections at various points in time, each intersection potentially contributing to the bouncing percept. When the timbres differed, however (Figure 1, middle and bottom panels), individual components did not cross as the $F_{0} \mathrm{~s}$ crossed.

2. The timbres were constant throughout each sound, so the subjects may have been influenced by the endpoints of the stimuli. They may, for instance, have given crossing responses when a sound with a particular timbre was heard to have a high initial pitch and a low final one. This cue to crossing $F_{0} s$ would only have been of use when the timbres were different.

In the present experiment, the possible confounds were addressed in the following ways. First, diphthong-like sounds, rather than different groups of harmonics, were used for the purpose of producing different timbres. All the "diphthongs" were composed of full harmonic series and, consequently, the corresponding harmonics from 
competing vowels all met in frequency simultaneously and at the same time that the $F_{0} \mathrm{~s}$ met. The behavior of both the harmonics and the $F_{0} \mathrm{~s}$ at the point of intersection was therefore maximally ambiguous for every stimulus. Second, each diphthong-like sound had differing initial and final timbres, which were not individually unique to that sound. In other words, all the diphthongs began with one of only four timbres and ended with another of those timbres, so a diphthong with a particular initial timbre could never be uniquely associated with either of the two final timbres in the stimulus.

In addition, the $F_{0} s$ of the constituent vowels had either continuous or inflected $F_{0}$ contours, so that some combinations of vowels had objectively rebounding $F_{0}$ s. As a result, the subjects had to use the continuity of timbre modulation at the $F_{0}$ intersection in order to discriminate crossing from bouncing pitch contours. The experiment was thus able not only to avoid any confounding influence of endpoint timbres, but also to test whether subjects make use of such endpoint cues, because, for stimuli in which one diphthong's initial timbre was the same as the other's final timbre, the use of such cues should be systematically misleading. For these stimuli, any exploitation of endpoint cues should prompt an incorrect response, depressing discrimination performance compared with conditions in which the endpoint cues are quite neutral.

\section{METHOD}

\section{Materials}

Formant trajectories for 12 "diphthongs" were derived from the formant frequencies of the steady-state vowels $/ \mathrm{i} /, / \mathrm{a} /, / \mathrm{u} /$, and $/ 3 /$ (from Assmann \& Summerfield, 1990), as shown in Table 1. Trajectories were produced by linear interpolation from each of the four vowel specifications to each of the other three (see Figure 2 for an example).

These diphthongs were synthesized by using an implementation of the Klatt (1980) synthesizer, which updated the $F_{0}$ and formant values for every $1 \mathrm{msec}$ of synthesized sound. The stimuli were $200 \mathrm{msec}$ in duration, and each had one of four $F_{0}$ contours: two were linear in $\log$ frequency and two were inflected at $100 \mathrm{msec}$. The four contours were characterized as "rising," ' falling," "ddipping," and "peaking"' (see Figure 3), and featured the following start, midpoint, and end values: rising, $80 \rightarrow 113.137 \rightarrow 160 \mathrm{~Hz}$; falling, $160 \rightarrow 113.137 \rightarrow 80 \mathrm{~Hz}$; dipping, $160 \rightarrow 113.137 \rightarrow$ $160 \mathrm{~Hz}$; peaking, $80 \rightarrow 113.137 \rightarrow 80 \mathrm{~Hz}$.

Rising and falling diphthongs were combined to make crossing stimuli and dipping and peaking diphthongs were combined to make bouncing stimuli. The two $F_{0}$ s present at a given point in a bouncing stimulus were thus always the same as those from the same point

Table 1

The Initial and Terminal Formant Frequencies (in Hertz) and 3-dB Bandwidths (in Hertz) Used to Synthesize the Individual Diphthongs

\begin{tabular}{cccccc} 
& \multicolumn{4}{c}{ Vowel } & \\
\cline { 2 - 5 } Formant & $/ \mathrm{i} /$ & \multicolumn{1}{c}{$/ \mathrm{a} /$} & $/ \mathrm{u} /$ & $/ 3 /$ & Bandwidth \\
\hline F1 & 250 & 650 & 250 & 450 & 90 \\
F2 & 2250 & 950 & 850 & 1250 & 110 \\
F3 & 3050 & 2950 & 1950 & 2650 & 170 \\
F4 & 3300 & 3300 & 3300 & 3300 & 250 \\
F5 & 3850 & 3850 & 3850 & 3850 & 300 \\
\hline
\end{tabular}

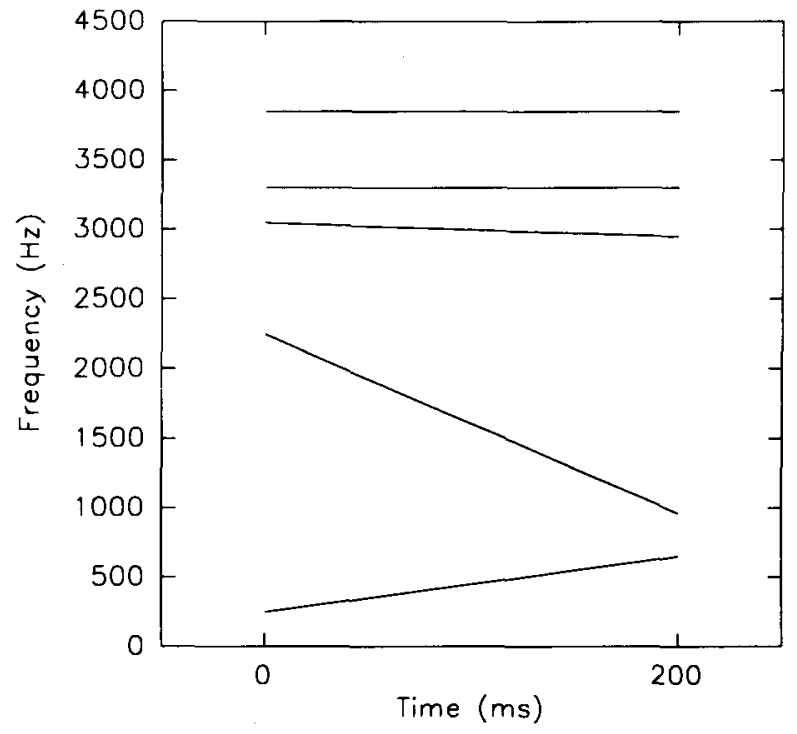

Figure 2. Example of formant movements for the diphthong-like sound; the timbre changes from $/ \mathrm{i} /-/ \mathrm{d} /$.

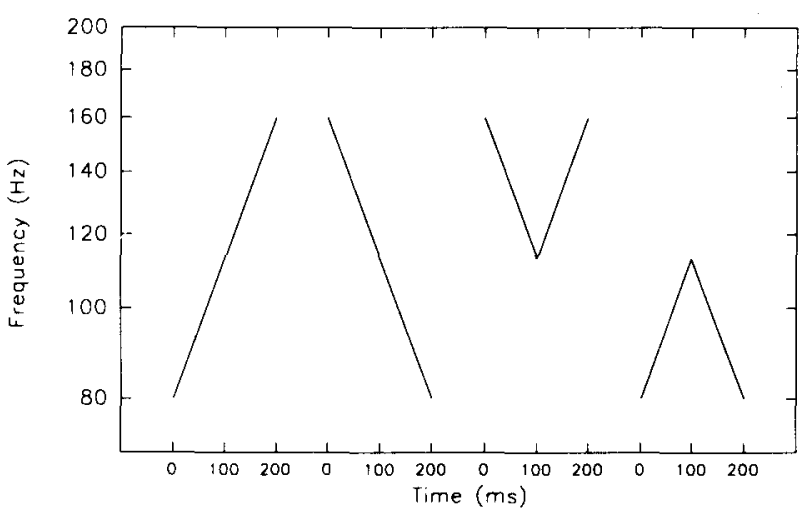

Figure 3. From left to right, respectively, the $F_{0}$ tracks for rising, falling, dipping, and peaking $F_{0}$ movements.

in a crossing stimulus. These two stimulus types were prepared by using each of the 132 exclusive combinations of the 12 diphthongs, making 264 stimuli altogether.

The 132 combinations were divided into five conditions, which are illustrated in Figure 4. The division was made according to whether or not the timbres of the two diphthongs differed at the point of $F_{0}$ intersection and, also, according to the correspondences that existed between the endpoint timbres of the two diphthongs. The stimuli of Condition I were the only ones to feature identical timbres at the $F_{0}$ intersection, so that the two timbres would, like the two $F_{0} \mathrm{~s}$, be perceived to either cross or bounce. Unless listeners (arbitrarily but "correctly") perceived the timbres as always to be crossing, they should be unable, in this condition, to discriminate between the two types of $F_{0}$ contour that were used in the synthesis. The five conditions were:

1. Crossing timbres (12 combinations). Each diphthong terminated with the timbre with which the other diphthong began (Figure $4 \mathrm{a}$ ). The equivalent crossing and bouncing stimuli consequently had identical timbres at the point of $F_{0}$ intersection and should not differ in their patterns of perceived $F_{0}$ movement. Bregman et al.'s (1982) findings suggest that these stimuli should evoke a bias toward bouncing responses. 

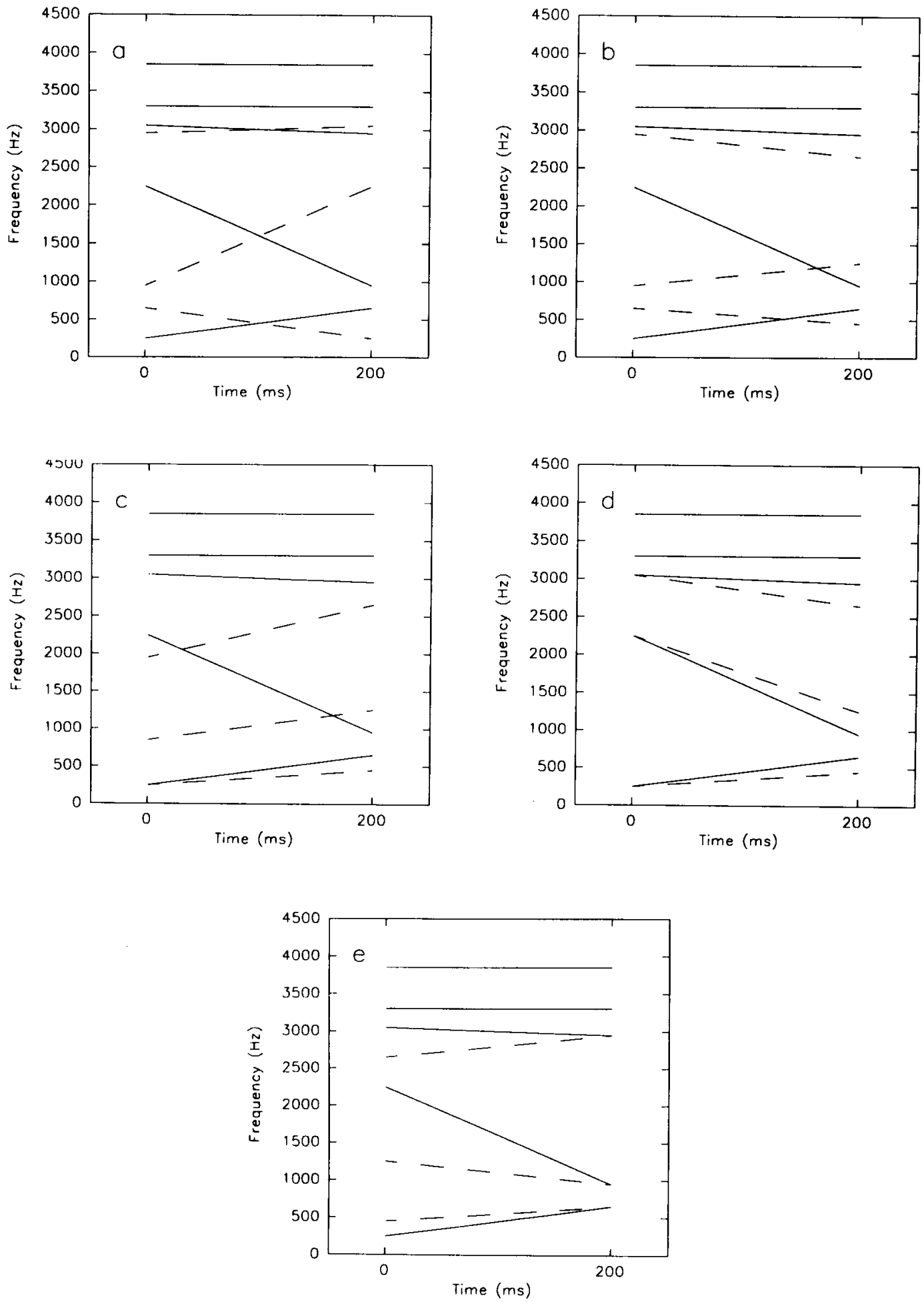

Figure 4. Examples of the formant tracks for two diphthongs (in each case, the tracks of the diphthong $/ \mathrm{i} / \rightarrow / \mathrm{a} /$ are in solid lines and those of the other diphthong are in dashed lines) for each of the five experimental conditions: (a) crossing timbres $(/ \mathrm{i} / \rightarrow / \mathrm{a} /$ and $/ \mathrm{a} / \rightarrow / \mathrm{i} /$ ), (b) returning timbre $(/ \mathrm{i} / \rightarrow / \mathrm{a} /$ and $/ \mathrm{a} / \rightarrow / 3 /)$, (c) different timbres $(/ \mathrm{i} / \rightarrow / \mathrm{a} /$ and $/ \mathbf{u} / \rightarrow / 3 /)$, (d) diverging timbres $(/ \mathbf{i} / \rightarrow / \mathrm{a} /$ and $/ \mathrm{i} / \rightarrow / 3 /)$, and (e) converging timbres $(/ \mathrm{i} /-/ \mathrm{a} /$ and $/ 3 / \rightarrow / a /)$. 
2. Different timbres (24 combinations). The four vowel specifications used to make the two diphthongs were all different (Figure $4 b$ ), so no correspondences existed between the endpoint timbres. The two timbres were different at the $F_{0}$ intersection, so crossing and bouncing stimuli may be discriminable.

3. Returning timbres (48 combinations). One diphthong terminated with the timbre with which the other diphthong began (Figure $4 \mathrm{c}$ ). The two timbres were different at the $F_{0}$ intersection, so crossing and bouncing stimuli may be discriminable, but, because one of the initial timbres swapped over to the competing diphthong at the end of a stimulus, any tendency for the subjects to associate similar initial and final timbres will lead them to make incorrect responses, and performance will be depressed compared with that in the different-timbres condition.

4. Diverging timbres (24 combinations). The two diphthongs had the same initial timbres, which diverged throughout the stimulus (Figure 4d). Again, the two timbres were different at the $F_{0}$ intersection, so crossing and bouncing stimuli may be discriminable.

5. Converging timbres ( 24 combinations). The two diphthongs had different initial timbres, which converged at the end (Figure 4e). The two timbres were different at the $F_{0}$ intersection, so crossing and bouncing stimuli may be discriminable.

The stimuli were played at a $10-\mathrm{kHz}$ sampling rate through a $12-$ bit digital-to-analog converter and were presented to the subjects over Sennheiser 414 headphones in a sound-attenuating booth at 85-90 dB(A). ${ }^{1}$

\section{Procedure}

Pilot experiments showed that the subjects varied markedly in their ability to discriminate between individual diphthongs that had inflected or uninflected $F_{0}$ s. Of the 7 subjects initially employed, 3 who were experienced in experiments using concurrent speech stimuli were able to make the required discrimination relatively well, and were retained for the two 40-min sessions described here.

Each session began with instructions that included a schematic representation of the four individual pitch contours. The subjects listened to the 48 individual diphthong sounds as a practice and attempted to differentiate "continuous" (falling or rising) from "inflected" (dipping or peaking) glides. No feedback was given during the practice.

The subjects then listened to the 264 experimental stimuli twice in a random order, and classified them as "crossing" or "bouncing" with a single keypress. Automatic feedback was given in the form of the correct response for the last trial.

\section{RESULTS}

\section{Practice Results}

The subjects' responses to the practice stimuli (continuous/inflected) were scored for each of the four types of glide, the continuous falling and rising glides, and the inflected dipping and peaking glides. The results are given in Table 2. From binomial probability, each of these scores deviated significantly from the chance score of $50 \%$ $(p<.000001)$.

Table 2

Percentage of Stimuli Correctly Identified as Continuous/Inflected Glides for Each Type of Practice Stimulus and Each Subject, Pooled Across Sessions

\begin{tabular}{ccc} 
& \multicolumn{2}{c}{ Percent Correct } \\
\cline { 2 - 3 } Subject & Continuous Glide & Inflected Glide \\
\hline L.M. & 56.25 & 64.58 \\
M.S. & 93.75 & 62.50 \\
H.C. & 66.68 & 66.67 \\
\hline
\end{tabular}

Table 3

Percent Correct, One-Tailed Probability and $d^{\prime}$ for Each Condition, Pooled Across Subjects

\begin{tabular}{lccc}
\hline \multicolumn{1}{c}{ Condition } & Percent Correct & \multicolumn{1}{c}{$p$} & \multicolumn{1}{c}{$d^{\prime}$} \\
\hline Crossing timbres & 47.9 & .720 & -0.15 \\
Different timbres & 65.5 & $<.000001$ & 0.80 \\
Returning timbre & 57.5 & $<.0002$ & 0.38 \\
Diverging timbres & 59.0 & $<.005$ & 0.53 \\
Converging timbres & 58.5 & $<.002$ & 0.49 \\
\hline
\end{tabular}

\section{Results for Each Condition}

Pooling across subjects and sessions, the crossingtimbres condition, in which crossing and bouncing stimuli were identical at the point of intersection, showed discrimination rates that did not differ from chance $(50 \%)$, whereas Conditions 2-5 all showed rates that were significantly above chance according to binomial probability (Table 3 ).

An analysis of variance for $d^{\prime}$ (Green \& Swets, 1966), covering the 3 subjects, the two repeated measures, and all five conditions, revealed significant differences between the conditions $[F(4,8)=9.95, p<.005]$. Tukey's post hoc analysis was used to compare the individual conditions. The crossing-timbres condition was found to give significantly lower $d$ 's than each of the other four conditions (different-timbres, $q=8.56, p<.01$; returningtimbre, $q=5.02, p<.05$; diverging-timbres, $q=5.98$, $p<.05$; converging-timbres, $q=6.13, p<.05$ ). No other pairwise comparisons were significant.

\section{Results for Individual Subjects}

Table 4 shows the number of correctly recognized $F_{0}$ contours from the main experiment for each subject, in each session, for each condition and each response category (crossing/bouncing). Also shown are the probabilities with which these scores (or higher) could arise by chance, derived from cumulative binomial probabilities, and also the $d^{\prime}$ and $\log _{e} \beta$ values (Green \& Swets, 1966), derived by treating crossing stimuli as signals to be detected.

Pooled across Conditions 2-5, for which effective crossing/bouncing discrimination was predicted, each subject performed significantly above chance in each session (Table 5).

\section{Response Biases}

In Condition 1, there was a large response bias toward bouncing responses, which constituted $79.9 \%$ of all responses to these stimuli. Unfortunately, $\beta$ is a poor measure of response bias in this instance, because the $\beta$ measure of response bias does not indicate a bias when the subjects could not perform the discrimination; when $d^{\prime}$ is zero, $\beta$ will also be zero.

Of the other four conditions (which provided timbrecontinuity cues), the different-timbres and returningtimbre conditions showed little bias $(54.7 \%$ and $44.2 \%$ crossing responses, respectively), but the convergingtimbres and diverging-timbres conditions showed a bias 
Table 4

For Each Session and Experimental Condition, Each Subject's Number of Correct Identifications for Each Stimulus Type in Each Session, Probabilities With Which These Scores (or Higher) Could Arise by Chance, and $d^{\prime}$ and $\log _{e} \beta$ Values

\begin{tabular}{|c|c|c|c|c|c|}
\hline \multirow{2}{*}{$\begin{array}{l}\text { Subject/ } \\
\text { Session }\end{array}$} & \multicolumn{2}{|c|}{ Correct Identifications } & \multirow[b]{2}{*}{$p$} & \multirow[b]{2}{*}{$d^{\prime}$} & \multirow[b]{2}{*}{$\log _{e}$} \\
\hline & Crossing & Bouncing & & & \\
\hline \multicolumn{6}{|c|}{ Crossing Timbres } \\
\hline L.M./1 & $2 / 24$ & $19 / 24$ & .844 & -0.57 & -6.3 \\
\hline L.M. $/ 2$ & $4 / 24$ & $17 / 24$ & .844 & -0.42 & -0.31 \\
\hline M.S./1 & $4 / 24$ & $21 / 24$ & .442 & 0.18 & 0.19 \\
\hline M.S. $/ 2$ & $2 / 24$ & $22 / 24$ & .557 & 0.00 & 0.00 \\
\hline H.C. $/ 1$ & $8 / 24$ & $15 / 24$ & .667 & -0.11 & -0.04 \\
\hline H.C. $/ 2$ & $6 / 24$ & $18 / 24$ & .557 & 0.00 & 0.00 \\
\hline \multicolumn{6}{|c|}{ Different Timbres } \\
\hline L.M./1 & $30 / 48$ & $29 / 48$ & $<.02$ & 0.58 & -0.02 \\
\hline L.M./2 & $36 / 48$ & $24 / 48$ & $<.01$ & 0.67 & -0.22 \\
\hline M.S./1 & $37 / 48$ & $30 / 48$ & $<.0002$ & 1.06 & -0.22 \\
\hline M.S. $/ 2$ & $31 / 48$ & $25 / 48$ & .0627 & 0.43 & -0.07 \\
\hline H.C. /1 & $28 / 48$ & $34 / 48$ & $<.005$ & 0.76 & 0.13 \\
\hline H.C. $/ 2$ & $40 / 48$ & $33 / 48$ & $<.0001$ & 1.46 & -0.34 \\
\hline \multicolumn{6}{|c|}{ Returning Timbre } \\
\hline L.M./I & $56 / 96$ & $58 / 96$ & $<.001$ & 0.58 & 0.00 \\
\hline L.M. $/ 2$ & $42 / 96$ & $64 / 96$ & .0851 & 0.47 & 0.08 \\
\hline M.S./1 & $55 / 96$ & $54 / 96$ & $<.05$ & 0.27 & 0.00 \\
\hline M.S. $/ 2$ & $45 / 96$ & $58 / 96$ & .174 & 0.19 & 0.03 \\
\hline H.C. $/ 1$ & $47 / 96$ & $62 / 96$ & $<.05$ & 0.35 & 0.07 \\
\hline H.C. $/ 2$ & $58 / 96$ & $63 / 96$ & $<.0005$ & 0.67 & 0.05 \\
\hline \multicolumn{6}{|c|}{ Diverging Timbres } \\
\hline L.M./1 & $34 / 48$ & $22 / 48$ & .0627 & 0.44 & -0.14 \\
\hline L.M. $/ 2$ & $33 / 48$ & $23 / 48$ & .0627 & 0.43 & -0.12 \\
\hline M.S./1 & $40 / 48$ & $20 / 48$ & $<.01$ & 0.76 & -0.45 \\
\hline M.S./2 & $33 / 48$ & $15 / 48$ & .541 & 0.00 & 0.00 \\
\hline H.C. $/ 1$ & $42 / 48$ & $17 / 48$ & $<.02$ & 0.79 & -0.60 \\
\hline H.C. $/ 2$ & $43 / 48$ & $15 / 48$ & $<.05$ & 0.77 & -0.67 \\
\hline \multicolumn{6}{|c|}{ Converging Timbres } \\
\hline L.M./1 & $32 / 48$ & $15 / 48$ & .620 & -0.06 & 0.03 \\
\hline L.M. $/ 2$ & $37 / 48$ & $19 / 48$ & .0627 & 0.48 & -0.24 \\
\hline M.S. $/ 1$ & $41 / 48$ & $19 / 48$ & $<.01$ & 0.79 & -0.53 \\
\hline M.S. $/ 2$ & $37 / 48$ & $21 / 48$ & $<.05$ & 0.59 & -0.26 \\
\hline H.C. $/ 1$ & $41 / 48$ & $21 / 48$ & $<.005$ & 0.90 & -0.55 \\
\hline H.C. $/ 2$ & $40 / 48$ & $17 / 48$ & $<.05$ & 0.59 & -0.40 \\
\hline
\end{tabular}

toward crossing responses $(69.6 \%$ and $70.1 \%$ crossing responses, respectively). This pattern of bias was also reflected in the $\log _{e} \beta$ measure. The former conditions gave small mean $\log _{e} \beta$ values of -0.12 and 0.04 , whereas the latter conditions indicated a bias toward crossing responses; in each case, mean $\log _{e} \beta=-0.33$.

\section{DISCUSSION AND CONCLUSIONS}

The results with the practice stimuli showed that the listeners could discriminate between the continuous and inflected pitch movements, even though the movements were quite rapid ( 5 octaves/sec) and were in the context of distracting timbre changes. Such abilities are demanded by normal speech; the $F_{0}$ contours plotted by O'Shaughnessy and Allen (1983), show that equally rapid $F_{0}$ movements are common, particularly on emphasized words, and that instantaneous reversals of the direction of move- ment, such as those employed in the present experiment, are often produced.

In the main experiment, the major comparison was between stimuli that did and did not have identical timbres at the point of $F_{0}$ intersection. As predicted, the subjects showed no discrimination ability when the timbres were the same at the $F_{0}$ intersection (Condition 1), whereas the subjects showed a modest, but highly significant, ability to differentiate the two classes of stimuli in Conditions 2-5. Because discrimination was reliable across Conditions 2-5, both when pooled across sessions and in three of six individual sessions, the effect is moderately robust and confirms Bregman et al.'s (1982) conclusion that continuity of timbre provides a cue to the disambiguation of $F_{0}$ intersections.

The subjects did not consistently discriminate between the two types of $F_{0}$ contour in Condition 1 (either "correctly" or "incorrectly"), so it does not seem that they employed any assumptions about timbre movements other than they do not "jump." That is to say, if the subjects were predisposed to hear timbre movements as uninflected and therefore crossing (perhaps by extrapolation from the rest of the stimuli in the experiment), then they should have been able to discriminate the $F_{0}$ movements "correctly." Given the opposite predisposition, they should have discriminated the stimuli, but labeled them "incorrectly."

Although the effect of continuity of timbre reported by Bregman et al. (1982) was genuine, their data could have been partially confounded by endpoint timbre cues. The present experiment offers only limited support for this suggestion because, for the different-timbres condition, which featured no correspondences between the timbres of the two diphthongs, performance was better than in the returning-timbre condition, in which such correspondences were systematically misleading, but this difference was not significant in the Tukey test $(q=3.54, p>.05)$.

The results from Condition 1 also confirm Bregman et al.'s (1982) finding that, when the timbres are the same at the point of $F_{0}$ intersection, there is a bias toward bouncing percepts. The preference for bouncing percepts in the present condition is much clearer than in Bregman et al.'s data; the subjects in the present study considered almost $80 \%$ of Condition 1 stimuli to be bouncing, whereas Bregman et al.'s data show only marginally higher rankings

Table 5

Percent Correct and Associated Binomial Probability for Each Subject in Each Session, Pooled Across Conditions 2-5

\begin{tabular}{lcl} 
Subject & Percent Correct & Probability \\
L.M. & Session 1 & \\
M.S. & 57.5 & $p<.001$ \\
H.C. & 61.7 & $p<.000001$ \\
& 60.4 & $p<.000001$ \\
L.M. & Session 2 & \\
M.S. & 57.9 & $p<.0005$ \\
H.C. & 55.2 & $p=.002$ \\
& 64.4 & $p<.000001$ \\
\hline
\end{tabular}


for inflection percepts in the equivalent condition. This result reinforces the suggestion that continuity of pitch movement is subordinate to the proximity of pitch before and after an $F_{0}$ intersection. The other observed response bias was somewhat unexpected; the two conditions in which two timbres diverged or converged elicited a crossing bias. A possible source of this effect may lie in formant tracking. The tracking of formant movements may produce effects that are analogous to the tracking of harmonics and $F_{0} \mathrm{~s}$. Because diphthongs of converging or diverging timbre have formants that do not intersect with each other (except where, for instance, one vowel's F1 intersects with the other's F2), these stimuli may be less likely to be perceived as having bouncing timbres. Hence, although the convergence or divergence of the formants may have little effect upon the perceived $F_{0}$ movements, they may, nonetheless, influence subjects' judgments by reducing the overall "bouncing" impression. Conversely, the simultaneous intersection of all the formant tracks in the crossing-timbres condition may be responsible for the strong bouncing bias in that condition.

Parsons's (1976) speech-separation algorithm achieved some success in dealing with $F_{0}$ intersections by exploiting good continuity of $F_{\mathbf{0}}$, but Stubbs and Summerfield (1990) implemented Parsons's algorithm and found that neither good continuity nor a variety of other heuristics based on $F_{0}$ were adequate at tracking $F_{0} \mathrm{~s}$ through intersections when competing voices were presented at similar levels. Stubbs and Summerfield concluded that constraints such as those of formant frequency and amplitude (i.e., timbre) must be required. The results of Bregman et al. (1982) and of the present study confirm that the human auditory system places emphasis on continuity of timbre rather than continuity of $F_{0}$ movement in interpreting an $F_{0}$ intersection. One reason for the auditory system's preferences may lie in the near-instantaneous $F_{0}$ inflections found in speech (O'Shaughnessy \& Allen, 1983), which might confuse a system that assumes continuity of movement.

In conclusion, the present experiment has unambiguously shown that timbre differences help listeners to follow the $F_{0}$ of a speaker's voice as it intersects with that of a competing voice. Thus, the results corroborate Bregman et al.'s (1982) earlier conclusion. Although performance levels were quite low, it should be remembered that the design of the experiment contained many features that made the task difficult; the changing-vowels timbres and rapid $F_{0}$ movements led to low performance, even in the practice session when diphthongs were presented singly. In real listening situations, timbres that are iden- tical at the point of $F_{0}$ intersection, diverging and converging timbres, and timbres exchanged between competing diphthongs would be rare. Instead, at least one timbre would, more likely, remain relatively constant. The effect of simply associating similar pre- and postintersection timbres, which may have partially confounded Bregman et al.'s results, would then supplement the effect demonstrated here.

\section{REFERENCES}

AssmanN, P. F., Sumerfield, Q. (1990). Modeling the perception of concurrent vowels: Vowels with different fundamental frequencies. Journal of the Acoustical Society of America, 88, 680-697.

Bregman, A. S., Halpern, L., Chalikia, M. H. (1982). Auditory partitioning of a set of tonal glides. Unpublished manuscript, McGill University, Department of Psychology, Montreal.

Brokx, J. P. L., Nootввоom, S. G. (1982). Intonation and the perceptual separation of simultaneous voices. Journal of Phonetics, 10 , 23-36.

Chalikia, M. H., de Bregman, A. S. (1989). The perceptual segregation of simultaneous auditory signals: Pulse train segregation and vowel segregation. Perception \& Psychophysics,46, 487-496.

Darwin, C. J., \& Culling, J. F. (1990). Speech perception seen through the ear. Speech Communication, 9, 469-476.

Green, D. M., \& Swets, J. A. (1966). Signal detection theory and psychophysics. New York: Wiley.

KLATT, D. H. (1980). Software for a cascade/parallel formant synthesizer. Journal of the Acoustical Society of America, 67, 838-844.

O'Shaughnessy, D., \& ALLEN, J. (1983). Linguistic modality effects on fundamental frequency in speech. Journal of the Acoustical Society of America, 74, 1155-1171.

PARSONS, T. W. (1976). Separation of speech from interfering speech by means of harmonic selection. Journal of the Acoustical Society of America, 60, 911-918.

SCHEFFERS, M. T. M. (1983). Sifting vowels: Auditory pitch analysis and sound segregation. Unpublished doctoral dissertation, Gronigen University.

StURBs, R. J., \& SUMmerfield, Q. (1990). Algorithms for separating the speech of interfering talkers: Evaluations with voiced sentences, and normal-hearing and hearing-impaired listeners. Journal of the Acoustical Society of America, 87, 359-372.

ZWICKER, U. T. (1984). Auditory recognition of diotic and dichotic vowel pairs. Speech Communication, 3, 265-277.

\section{NOTE}

1. These figures are based upon the levels of isolated static vowels, synthesized according to Assmann and Summerfield's (1990) parameters. When presented with the current experimental setup, the levels for these static vowels fall between 77 and $89 \mathrm{~dB}(\mathrm{~A})$. The level of a complete dynamic stimulus is somewhat variable, because it depends, at any point in time, upon the current timbres of each of the two constituent diphthongs and their degree of glottal pulse synchrony. Both of these factors change continuously during a stimulus.

(Manuscript received October 7, 1992; revision accepted for publication February 22, 1993.) 